\title{
Association of Serum Biomarkers With Pulmonary Involvement of Rheumatoid Arthritis Interstitial Lung Disease: From KORAIL Cohort Baseline Data
}

Jinyoung Moon, M.D. ${ }^{1}$, Jeong Seok Lee, M.D. ${ }^{2}$, Young $\operatorname{Im}$ Yoon, R.N. ${ }^{3}$, Sung Hae Chang, M.D. ${ }^{4}$, Yeon Ah Lee, M.D. ${ }^{5}$, You-Jung Ha, M.D. ${ }^{6}$, Eun Ha Kang, M.D., Ph.D. ${ }^{6}$, Yong-Beom Park, M.D., Ph.D. ${ }^{7}$, Hwajeong Lee, M.D. ${ }^{8}$, Jung-Yoon Choe, M.D., Ph.D. ${ }^{8}$, Eun Young Lee, M.D., Ph.D. ${ }^{1,3}$; Study Group, Korean Rheumatoid Arthritis Interstitial Lung Disease (KORAIL)

${ }^{1}$ Division of Rheumatology, Department of Internal Medicine, Seoul National University College of Medicine, Seoul, ${ }^{2}$ Graduate School of Medical Science and Engineering, Korea Advanced Institute of Science and Technology, Daejeon, ${ }^{3}$ Division of Rheumatology, Department of Internal Medicine, Seoul National University Hospital, Seoul, ${ }^{4}$ Division of Rheumatology, Department of Internal Medicine, Soonchunhyang University College of Medicine, Cheonan, ${ }^{5}$ Division of Rheumatology, Department of Internal Medicine, School of Medicine, Kyung Hee University, Seoul, ${ }^{6}$ Division of Rheumatology, Department of Internal Medicine, Seoul National University Bundang Hospital, Seongnam, ${ }^{7}$ Division of Rheumatology, Department of Internal Medicine, Yonsei University College of Medicine, Seoul, ${ }^{8}$ Division of Rheumatology, Department of Internal Medicine, Daegu Catholic University School of Medicine, Daegu, Korea

Objective. The increase in mortality in rheumatoid arthritis (RA) patients with interstitial lung disease (ILD) is well known. However, there are few studies on serum markers that can evaluate acute exacerbation or prognosis in RA-ILD patients. The purpose of this study was to identify the association between biomarkers and lung lesions in patients with RA-ILD. Methods. We analyzed 153 patients with serum samples in a prospective, multicenter cohort of Korean RA-ILD patients. The serum levels of biomarkers, matrix metalloproteinase (MMP-7), surfactant protein-D (SP-D), and Krebs von den Lungen-6 (KL-6) were measured and correlated with forced vital capacity (FVC), diffusing capacity for carbon monoxide (DLCO) and the results of computed tomography $(\mathrm{CT})$. CT results were interpreted semi-quantitatively according to the extent of lung lesions (grade $1,0 \% \sim$ $25 \%$; grade 2, 26\% 50\%; grade 3, 51\% 75\%; grade 4, 76\% 100\%). Results. MMP-7, SP-D, and KL-6 were negatively correlated with FVC (MMP-7, $r=-0.267, p=0.001 ;$ SP-D, $r=-0.250, p=0.002 ; K L-6, r=-0.223, p=0.006)$ and DLCO (MMP-7, $r=-0.404, p<0.001 ;$ SP-D, $r=-0.286, p=0.001 ; K L-6, r=-0.226, p=0.007)$. In addition, MMP-7, SP-D, and KL-6 tended to increase with higher grades of lung lesions on CT (MMP-7, $p=0.013 ; S P-D, p<0.001 ; K L-6, p<0.001)$. Conclusion. MMP-7, SP-D, and KL-6 can be used to evaluate the functional and anatomical status of lung involvement in the RA-ILD patients. (J Rheum Dis 2021;28:234-241)

Key Words. Rheumatoid arthritis with interstitial lung disease, Matrix metalloproteinase-7, Surfactant protein-D, Krebs von den Lungen-6

\section{INTRODUCTION}

Rheumatoid arthritis (RA) has many extra-articular manifestations [1]. The prevalence of interstitial lung disease (ILD) in RA patients has been reported to range from $1 \% \sim 58 \%$, showing a large difference between studies [2]. This differs according to the definition and diagnostic method of ILD. Clinically evident RA-ILD occurs in approximately $10 \%$ of patients with RA $[2,3]$. Subclinical disease (no previous ILD diagnosis but interstitial lung abnormalities on computed tomography [CT]) is present in an additional $30 \%$ of individuals [3-5], with 34\% $57 \%$ demonstrating radiologic progression over 1 to 2 years $[6,7]$. Despite the development of drugs that con-

Received : April 23, 2021, Revised : May 31, 2021, Accepted : June 3, 2021

Corresponding to : Eun Young Lee (iD http://orcid.org/0000-0001-6975-8627

Division of Rheumatology, Department of Internal Medicine, Seoul National University College of Medicine, 103 Daehak-ro, Jongno-gu, Seoul 03080, Korea. E-mail : elee@snu.ac.kr 
trol articular manifestations of RA [8], ILD is the only complication of RA that is increasing in prevalence $[1,2]$. Furthermore, the mortality associated with RA-ILD has increased [3].

The pathogenesis of RA-ILD is not clear; however, it is known to have a mechanism similar to that of idiopathic pulmonary fibrosis (IPF). Dysregulated inflammatory processes promote the production of cytokines, chemokines, and growth factors, causing a series of changes. This eventually results in epithelial cell damage, angiogenesis, fibroblast differentiation, and lung fibrosis [9]. Although the association of serum biomarkers with RA-ILD is not clear, it has been established that tumor necrosis factor alpha (TNF- $\alpha$ ) and interleukin 6 (IL-6) are one of the several cytokines related to RA [10].

Previous studies have shown that serum Krebs von den Lungen 6 (KL-6) levels are associated with ILD [11-13], which is associated with mortality [14]. A recent study found that serum KL-6 is related to the severity of connective tissue disease-associated interstitial lung disease (CTD-ILD) [15]. Other recent studies showed that when KL-6 was elevated above a certain level, the frequency of the usual interstitial pneumonia (UIP) pattern among RA-ILD was high [16], and it was also related to mortality $[16,17]$. In addition, when compared with the RA-nonILD group, the KL-6 level was elevated in the RA-ILD group, and positive correlation with the fibrosis score of chest CT was also proven [18].

Although not clear, RA-ILD, especially RA-UIP, and IPF are known to have many histopathological and clinical aspects in common. IPF is a chronic and progressive disorder of extracellular matrix (ECM) remodeling. In previous studies, matrix metalloproteinase (MMP)- 7 and MMP-1, known as ECM-modulating enzymes, have been shown to be potential biomarkers of IPF [19]. In addition to MMP-7, surfactant protein-D (SP-D) levels are elevated in IPF patients [20]. Another study revealed that MMP-7 could be used as a biomarker in patients with RA-ILD [21]. In addition, a study showed that MMP-7 and SP-D are related to RA-ILD, as well as risk factors (age, sex, smoking, rheumatoid factor, and anticyclic citrullinated peptide antibodies) known in RA patients [22]. Another recent study showed that the SP-D level was significantly elevated in the RA-ILD group when compared with control and RA-non-ILD groups [23].

Based on the results of these existing studies, we have derived the following hypotheses. First, can serum KL-6, MMP-7, and SP-D be used as biomarkers to evaluate acute exacerbation in RA-ILD patients? Most of the existing studies have focused on the difference in biomarker levels between the RA group and the RA-ILD group. We tried to evaluate the usefulness of biomarkers in situations where pulmonary function test (PFT) or chest CT cannot be performed when acute exacerbation occurs in patients with already confirmed RA-ILD. Second, are TNF-alpha and IL-6, which are known to be related to RA, also associated with lung lesions in RA-ILD patients? This is a question of whether the disease mechanism of RA-ILD can be evaluated in line with the joint involvement of RA.

For the reasons described above, the correlation between serum levels of markers (IL-6, TNF- $\alpha$, KL-6, MMP-7 and SP-D) and diffusing capacity for carbon monoxide (DLCO) and forced vital capacity (FVC) measured by PFT in the RA-ILD cohort was analyzed. In addition, the trend of biomarkers and cytokines was evaluated when $\mathrm{CT}$ grade increased.

\section{MATERIALS AND METHODS}

\section{Study population}

The KOrean Rheumatoid Arthritis Interstitial Lung Disease (KORAIL) group is a multicenter, prospective cohort study developed to help understand the disease characteristics of RA patients with ILD. Six centers in South Korea (Daegu Catholic University Medical Center, Kyung Hee University Hospital, Seoul National University Hospital, Seoul National University Bundang Hospital, Severance Hospital, and Soonchunhyang University Hospital) participated in patient enrollment and follow-up. Patient enrollment was performed between May 2015 and June 2018. The inclusion criteria included definite RA (score 26 ) classified according to the American College of Rheumatology (ACR)/European League Against Rheumatism (EULAR) 2010 Rheumatoid Arthritis Classification Criteria. ILD was confirmed using high-resolution computed tomography (HRCT) and PFTs.

\section{Ethical consideration}

This study was performed in compliance with the Declaration of Helsinki, and the Institutional Review Boards (IRB) at the six centers approved the study (IRB numbers; CR-15-009, Daegu Catholic University Medical Center; 2016-11-063, Kyung Hee University Hospital; 1407-027-592, Seoul National University Hospital; B-1412-280-412, Seoul National University Bundang 
Hospital; 2014-2417-002, Severance Hospital; 2016-01-008, Soonchunhyang University Hospital). Written informed consent was obtained from all the patients.

\section{Measurements}

Baseline demographic data (age, sex, body mass index, and smoking status) were collected at the time of enrollment. The date of RA diagnosis and the date of diagnosis of ILD were reviewed to estimate disease duration. RA disease activity was evaluated by the Disease Activity Score 28 (DAS28) and a subjective method using the Health Assessment Questionnaire-Disability Index (HAQ-DI) and the Patient Global Assessment (PGA). Medications prescribed after the diagnosis of ILD were reviewed. DLCO and FVC measured by PFT, 6 min walk test (6 MWT), and HRCT were performed within 6 months preceding or following the first visit. If there was a test that was evaluated within 6 months before enrollment, it was not re-measured at the time of enrollment. In addition, when the appointment of the examination was not available or an immediate examination was difficult due to the patient's personal reasons, the examination was performed within 6 months from the time of enrollment. Laboratory data such as rheumatoid factor (RF) positivity and titer and anti-cyclic citrullinated peptide antibody (anti-CCP) positivity and titer were obtained by medical chart review.

\section{Serum parameters}

Baseline serum sampling was performed during the first visit. TNF- $\alpha$, IL-6, KL-6, MMP-7, and SP-D were evaluated.

Cytokine levels were measured using the MSD multi-spot assay system (Meso Scale Discovery, Gaithersburg, MD, USA). TNF- $\alpha$ and IL- 6 levels were measured by human proinflammatory panel I (V-Plex). KL-6 levels were measured with the Nanopia KL-6 assay (Sekisui Medical Co., LTD, Tokyo, Japan) using the latex-enhanced immunoturbidimetric assay method. MMP-7 and SP-D levels were measured using MSD R-PLEX assays (Meso Scale Discovery).

\section{Review of ILD on HRCT}

One trained rheumatologist graded the ILD extent of HRCT semi-quantitatively (grade 1, $0 \% \sim 25 \%$; grade 2, $26 \% \sim 50 \%$; grade $3,51 \% \sim 75 \%$; grade $4,75 \% \sim 100 \%$ ) [15]. If lung lesions apart from ILD were discovered, the CT scan was excluded from the analysis.
Table 1. Baseline characteristics of patients obtained serum sample $(n=153)$

\begin{tabular}{|c|c|}
\hline Variable & Value \\
\hline \multicolumn{2}{|l|}{ Demographic parameters } \\
\hline Age $(y r)$ & $66.35 \pm 8.22$ \\
\hline Sex, female & 107 (69.9) \\
\hline Body mass index & $23.40 \pm 2.95$ \\
\hline Ever smoker & $39(25.5)$ \\
\hline Pack-years of smoking & $32.05 \pm 10.88$ \\
\hline \multicolumn{2}{|l|}{ RA parameters } \\
\hline $\mathrm{RF}(\mathrm{IU} / \mathrm{mL})$ & $244.24 \pm 500.53$ \\
\hline Anti-CCP (units/mL) & $199.62 \pm 166.86$ \\
\hline DAS28-ESR & $3.99 \pm 1.47$ \\
\hline DAS28-CRP & $3.10 \pm 1.42$ \\
\hline HAQ-DI & $0.68 \pm 0.76$ \\
\hline PGA* & $36.41 \pm 26.38$ \\
\hline Duration of RA (yr) & $8.08 \pm 8.14$ \\
\hline \multicolumn{2}{|l|}{ Medication use (ever) } \\
\hline Glucocorticoids & $145(94.8)$ \\
\hline Methotrexate & $99(64.7)$ \\
\hline Hydroxychloroquine & $98(64.1)$ \\
\hline Sulfasalazine & $91(59.5)$ \\
\hline Leflunomide & $57(37.3)$ \\
\hline Tacrolimus & $49(32.0)$ \\
\hline Etanercept & $21(13.7)$ \\
\hline Infliximab & $8(5.2)$ \\
\hline Adalimumab & $11(7.2)$ \\
\hline Tocilizumab & $10(6.5)$ \\
\hline Abatacept & $13(8.5)$ \\
\hline \multicolumn{2}{|l|}{ Pulmonary function test } \\
\hline FEV1, \% of predicted & $92.26 \pm 21.52$ \\
\hline FVC, \% of predicted & $85.56 \pm 16.60$ \\
\hline DLCO, $\%$ of predicted & $71.89 \pm 19.47$ \\
\hline Six minute walk test, meter & $342.71 \pm 112.11$ \\
\hline \multicolumn{2}{|l|}{ CT grade } \\
\hline Grade $1(0 \% \sim 25 \%)$ & $86(56.2)$ \\
\hline Grade $2(26 \% \sim 50 \%)$ & $46(30.1)$ \\
\hline Grade $3(51 \% \sim 75 \%)$ & $15(9.8)$ \\
\hline Grade $4(75 \% \sim 100 \%)$ & $6(3.9)$ \\
\hline \multicolumn{2}{|l|}{ Serum levels } \\
\hline TNF- $\alpha(p g / m L)$ & $3.46 \pm 8.54$ \\
\hline IL-6 (pg/mL) & $6.09 \pm 24.31$ \\
\hline MMP-7 (pg/mL) & $7,524.35 \pm 4,481.77$ \\
\hline SP-D (pg/mL) & $9,721.65 \pm 7,148.10$ \\
\hline KL-6 (units/mL) & $598.35 \pm 545.76$ \\
\hline
\end{tabular}

Values are presented as mean \pm standard deviation or number (\%). RA: rheumatoid arthritis, RF: rheumatoid factor, Anti-CCP: anti-cyclic citrullinated peptide antibody, DAS28: disease activity score of 28 joints, ESR: erythrocyte sedimentation rate, CRP: C-reactive protein, HAQ-DI: health assessment questionnairedisability index, PGA: patient global assessment, FEV1: forced expiratory volume in one second, FVC: forced vital capacity, DLCO: diffusing capacity for carbon monoxide, TNF- $\alpha$ : tumor necrosis factor $\alpha$, IL-6: interleukin 6, MMP-7: matrix metallopeptidase 7, SP-D: surfactant protein D, KL-6: Krebs von den Lungen 6. *PGA ranges from 0 to $100 \mathrm{~mm}$. 


\section{Statistical analysis}

The baseline statistics (mean, standard deviation, and percentage) of each variable were calculated. The Spearman's correlation coefficient was used to analyze the association between serum TNF- $\alpha$, IL-6, KL-6, MMP-7, and SP-D levels and DLCO and FVC. Through univariable analysis, variables with significant association with FVC and DLCO were controlled and multivariable analysis was performed. The p-value for trend was analyzed to evaluate the difference in serum cytokine and biomarker levels between the CT grade groups. Statistical significance was set at $\mathrm{p}<0.05$. The data was analyzed using SPSS Statistics software, version 26.0 (IBM Co., Armonk, NY, USA).

\section{RESULTS}

\section{Baseline characteristics of the KORAIL cohort}

Table 1 shows the baseline characteristics of 153 individuals included in the analysis, including disease characteristics of both RA and ILD. The mean age was $66.35 \pm 8.22$ years and $69.9 \%$ were female. Of these, $25.5 \%$ were ever smokers. The mean values of RF and anti-CCP were 244.24 $\pm 500.53 \mathrm{IU} / \mathrm{mL}$ and 199.62 \pm 166.86 units/mL, respectively. The mean duration of RA was
$8.08 \pm 8.14$ years and the mean duration of ILD was $2.88 \pm 3.15$ years. DAS28-ESR was $3.99 \pm 1.47$ and DAS28-CRP was $3.10 \pm 1.42$ showing moderate disease activity of RA in this cohort.

Patients' medical treatment history are also introduced in Table 1. Glucocorticoids were used in $94.8 \%$ of the patients. Methotrexate, hydroxychloroquine, and sulfasalazine were used in approximately $60 \%$ of patients. Leflunomide and tacrolimus were used in $37.3 \%$ and $32.0 \%$ of patients, respectively. Etanercept, infliximab, and adalimumab were used in $13.7 \%, 5.2 \%$, and $7.2 \%$ of patients, respectively.

The mean value of FVC was in the normal range $(85.56 \pm 16.60 \%)$, and the mean value of DLCO was slightly decreased $(71.89 \pm 19.47 \%)$ compared to the normal reference value. In semiquantitative CT grade evaluation, grade 1 was $56.2 \%$, grade 2 was $30.1 \%$, grade 3 was $9.8 \%$, and grade 4 was $3.9 \%$.

\section{Correlations between FVC and DLCO and serum biomarkers}

In univariable analysis, age, DAS28-ESR, and DAS28CRP showed correlations with FVC. Sex, DAS28-ESR, DAS28-CRP, and HAQ also showed correlations with DLCO (Supplementary Table 1). These variables were
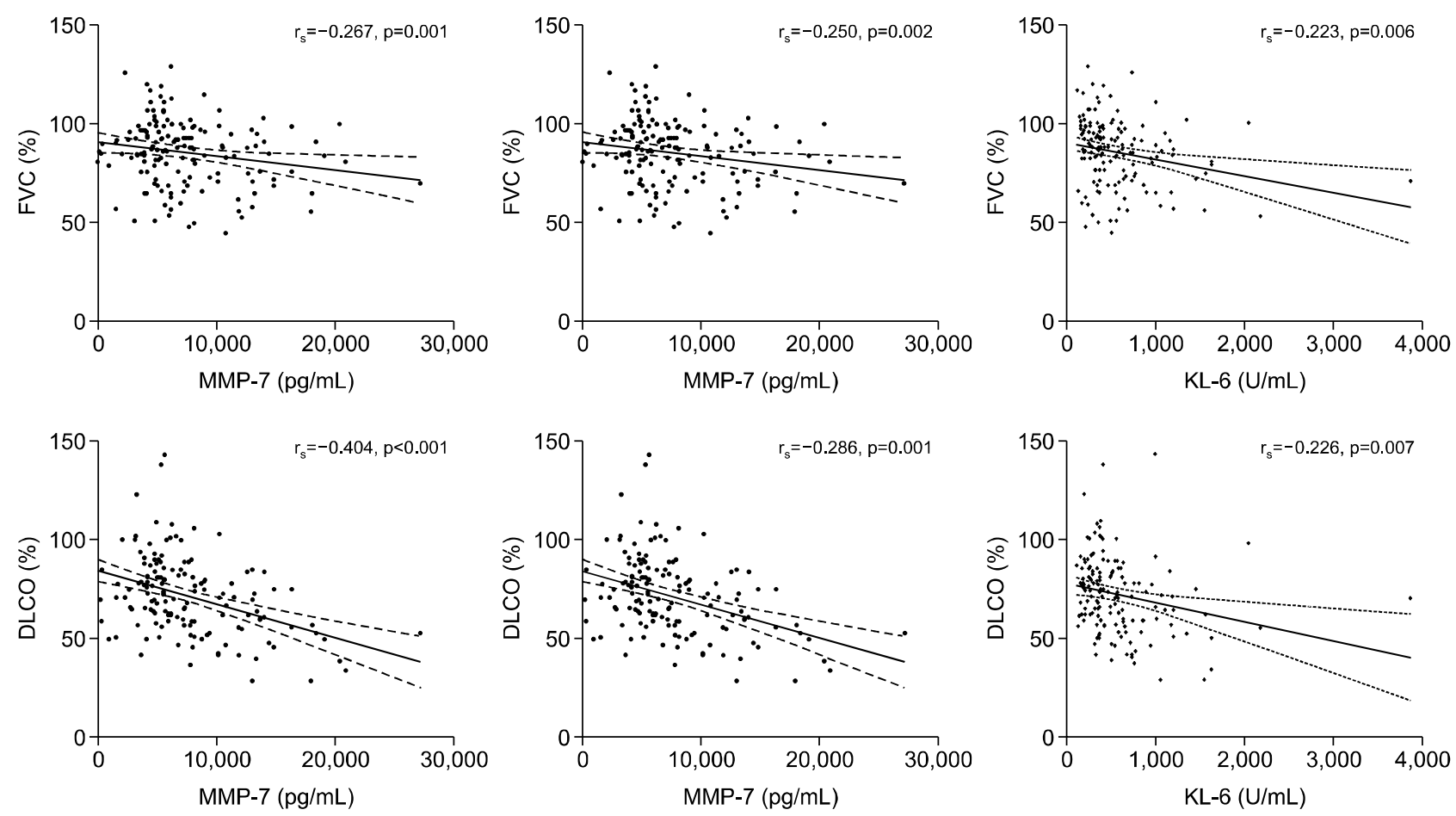

Figure 1. Correlations between FVC and DLCO and serum MMP-7, SP-D and KL-6. FVC: forced vital capacity, DLCO: diffusing capacity for carbon monoxide, MMP-7: matrix metallopeptidase 7, SP-D: surfactant protein D, KL-6: Krebs von den Lungen 6. 
Jinyoung Moon et al.

controlled, and multivariable analysis was performed. Serum MMP-7 levels were negatively correlated with FVC $(\mathrm{r}=-0.267, \mathrm{p}=0.001)$ and DLCO $(\mathrm{r}=-0.404, \mathrm{p}<$ 0.001). In addition, serum SP-D levels were also negatively correlated with FVC $(r=-0.250, p=0.002)$ and $\operatorname{DLCO}(r=-0.286, p=0.001)$. This correlation was also observed for KL-6 levels, it was negatively correlated with FVC $(r=-0.223, p=0.006)$ and DLCO $(r=-0.226$, $\mathrm{p}=0.007)$. TNF- $\alpha$ and IL-6 levels showed no correlation with FVC and DLCO (Figure 1 and Table 2).

\section{Serum biomarker levels according to semiquantitative $\mathrm{CT}$ grades}

MMP-7, SP-D, and KL-6 levels tended to increase with the increasing CT grade (MMP-7, $\mathrm{p}=0.013$; SP-D, $\mathrm{p}<$ 0.001; KL-6, $\mathrm{p}<0.001$ ). TNF- $\alpha$ and IL-6 levels did not show this trend (Figure 2 and Table 3 ).

\section{Correlations between 6MWT and serum biomarkers}

The correlation between 6MWT and cytokines and biomarkers was analyzed. There were no significant results for all measurements (TNF- $\alpha, r=-0.023, \mathrm{p}=0.789$;

Table 2. Correlations between FVC and DLCO and serum cytokines and biomarkers

\begin{tabular}{|c|c|c|c|c|c|}
\hline & TNF- $\alpha$ & IL-6 & MMP-7 & SP-D & KL-6 \\
\hline \multicolumn{6}{|l|}{ FVC } \\
\hline$r_{\mathrm{s}}$ & -0.074 & -0.085 & -0.267 & -0.250 & -0.223 \\
\hline p-value & 0.368 & 0.305 & 0.001 & 0.002 & 0.006 \\
\hline \multicolumn{6}{|l|}{ DLCO } \\
\hline$r_{s}$ & -0.127 & -0.063 & -0.404 & -0.286 & -0.226 \\
\hline p-value & 0.131 & 0.454 & 0.000 & 0.001 & 0.007 \\
\hline
\end{tabular}

FVC: forced vital capacity, DLCO: diffusing capacity for carbon monoxide, TNF- $\alpha$ : tumor necrosis factor $\alpha$, IL-6: interleukin 6, MMP-7: matrix metallopeptidase 7, SP-D: surfactant protein D, KL-6: Krebs von den Lungen 6.
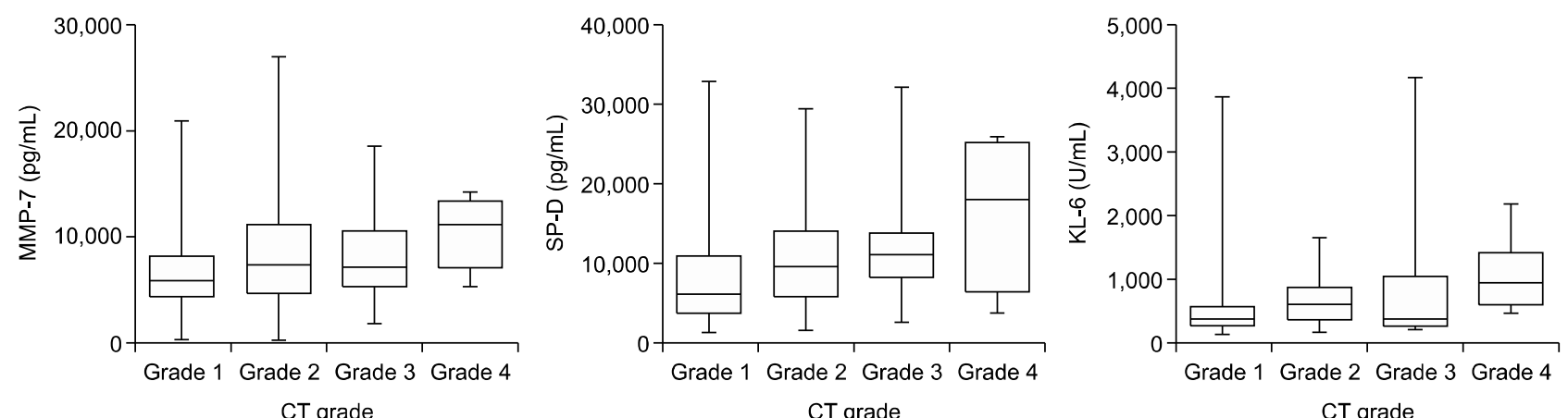

Figure 2. Serum MMP-7, SP-D, and KL-6 levels according to semiquantitative CT grades. MMP-7: matrix metallopeptidase 7, SP-D: surfactant protein D, KL-6: Krebs von den Lungen 6, CT: computed tomography.

Table 3. Serum TNF- $\alpha$, IL-6, MMP-7, SP-D, and KL-6 levels according to semiquantitative CT grades

\begin{tabular}{lcccrr}
\hline \hline & Grade $1(\mathrm{n}=86)$ & Grade $2(\mathrm{n}=46)$ & Grade $3(\mathrm{n}=15)$ & Grade $4(\mathrm{n}=6)$ & $\mathrm{p}$-value \\
\hline TNF- $\alpha(\mathrm{pg} / \mathrm{mL})$ & $3.26 \pm 8.46$ & $4.35 \pm 9.98$ & $2.55 \pm 5.69$ & $1.72 \pm 0.67$ & 0.674 \\
IL-6 $(\mathrm{pg} / \mathrm{mL})$ & $4.50 \pm 13.69$ & $10.33 \pm 40.13$ & $3.43 \pm 3.72$ & $3.16 \pm 2.03$ & 0.282 \\
MMP-7 $(\mathrm{pg} / \mathrm{mL})$ & $6,885.47 \pm 3,890.06$ & $8,074.50 \pm 5,342.70$ & $8,361.50 \pm 4,730.29$ & $10,396.35 \pm 3,465.75$ & 0.013 \\
SP-D $(\mathrm{pg} / \mathrm{mL})$ & $8,422.91 \pm 6,899.35$ & $10,399.37 \pm 6,518.88$ & $12,472.11 \pm 7,794.94$ & $16,264.79 \pm 9,174.95$ & $<0.001$ \\
KL-6 $(\mathrm{units} / \mathrm{mL})$ & $485.84 \pm 471.36$ & $675.98 \pm 385.39$ & $824.20 \pm 1016.52$ & $1,051.10 \pm 612.84$ & $<0.001$ \\
\hline
\end{tabular}

Values are presented as mean \pm standard deviation. TNF- $\alpha$ : tumor necrosis factor $\alpha$, IL-6: interleukin 6, MMP-7: matrix metallopeptidase 7, SP-D: surfactant protein D, KL-6: Krebs von den Lungen 6, CT: computed tomography. 
IL-6, $r=-0.110, p=0.201$; MMP-7, $r=-0.153, p=0.074$; SP -D, $r=0.085, p=0.319 ; \mathrm{KL}-6, r=0.012, \mathrm{p}=0.890$ ) (Supplementary Table 2).

\section{Correlations between KL-6, MMP-7, and SP-D}

The correlation coefficient between KL-6 and MMP-7 was $0.294(p=0.01)$, between KL-6 and SP-D was 0.460 $(\mathrm{p}=0.01)$, and between MMP-7 and SP-D was 0.199 $(p=0.014)$.

\section{DISCUSSION}

In this study, we investigated the association of serum cytokines and biomarkers with FVC, DLCO, and CT grade in the KORAIL group.

The life expectancy of patients with RA is reportedly shorter by $10 \sim 11$ years compared to the general population [24]. ILD is a major cause of death [2,3]. RA patients with ILD are known to have a 3 times higher risk of death than RA patients without ILD. One study of Korean RA patients showed a significant increase in mortality with ILD (hazard ratio [HR]: 7.89, confidence interval [CI]: $3.16 \sim 19.69, \mathrm{p}<0.01$ ), which appeared to be higher than those with cardiovascular disease. (HR: 4.10, CI: $1.79 \sim 9.37, \mathrm{p}<0.01$ ) [25]. In previous studies, the prevalence of ILD in Korean RA patients through CT scan was reported to be $9.8 \% \sim 12.6 \%[25,26]$. This is a high frequency comorbidity in RA patients, and thus we have constructed the Korean RA-ILD patients' cohort. This cohort has advantages in that serum sampling, PFT, and CT could be performed at approximately the same time. We attempted to confirm the relationship between PFTs, CT results, and cytokine and biomarker levels in patients with RA-ILD. This can be useful in situations where PFT or CT cannot be performed due to acute exacerbation of pulmonary conditions. In addition, it is difficult to perform CT scans frequently because of radiation hazards and a problem of reservation schedule. In this case, using biomarkers to predict lung conditions may be helpful.

Representative cytokines in previous studies of RA include TNF- $\alpha$ and IL-6 [27-29], and the target drug for these cytokines is widely used in clinic. For this reason, the relationship between severity of ILD evaluated by FVC and DLCO and cytokines was analyzed, but the association was limited. TNF- $\alpha$ and IL-6 levels were not statistically different according to the CT grade. The association between RA-ILD and cytokines, known to be associated with RA, is an inconsistent finding. This suggests that RA-ILD is a separate disease entity from RA. In fact, it can be seen that the ILD worsens after using disease-modifying anti-rheumatic drugs in clinical settings. This fact supports our hypothesis. Further study is needed for this hypothesis in the future.

MMP-7 has been found to be associated with severity of ILD [21]. A previous study showed that MMP-7 and SP-D are predictors of RA-ILD [22]. In this study, MMP-7 and SP-D showed differences between the group of RA patients without ILD and the subclinical and clinically evident RA-ILD group. KL- 6 has been found to be associated with the severity and mortality of ILD [11-13,15]. The association between MMP-7 and numerical score based on the type and distribution of interstitial lung abnormalities on chest CT has been demonstrated in patients with RA-ILD [21]. The association between KL-6 and semiquantitative CT grade has been demonstrated in patients with CTD-ILD [15] and in patients with RA-ILD [30]. In our study, the results were consistent with previous studies. MMP-7, SP-D, and KL-6 levels were negatively correlated with both FVC and DLCO. MMP-7, SP-D, and KL-6 showed a tendency to increase as semiquantitative CT grades increased. In patients with IPF, MMP-7 and SP-D are known to predict disease progression and survival [19,31]. Its clinical significance in patients with RA-ILD may be revealed through cohort follow-up. It is expected that future data will be able to predict disease progression of RA-ILD based on the initial biomarker levels. In previous studies, it was found that the frequency of UIP among RA-ILD increased according to KL-6 level, and this was related to mortality [16].

Our study has several limitations. First, there is no control group. In contrast to the previous studies comparing RA and RA-ILD patients to evaluate prognosis and to analyze the association with serum markers, our study subjects do not include the RA-non-ILD group, which has limitations. Second, the cohort group was targeted, but the cross-sectional data at the time of enrollment was used for analysis. However, we continue to secure data and plan to analyze using longitudinal data in the future. The number of RA-ILD patients alone was 153, which is a relatively large sample size considering the number of patients in existing studies. Third, patient's subjective symptom index (e.g., Medical Research Council dyspnea score) was not collected for ILD. This indicator may be particularly useful in early ILDs where radiographic lung involvement is not severe, but for practical reasons, it was difficult to collect the data at that point. 


\section{CONCLUSION}

In the RA-ILD cohort, MMP-7, SP-D, and KL-6 levels were negatively correlated with FVC and DLCO. Furthermore, MMP-7, SP-D, and KL-6 were correlated with the semiquantitative grade of CT. These biomarkers can be used to evaluate the functional and anatomical status of lung involvement in RA-ILD.

\section{ACKNOWLEDGMENTS}

We are so thankful to Seon Uk Kim, Ji Hye Lee, and Ji Yong Choi at Center for Medical Innovation, Biomedical Research Institute, and Seoul National University hospital for their efforts on large sample preparation and measurement of serum markers and cytokines.

This work was supported by Seoul National University Hospital (Cohort project No. 2520160060) and SEKISUI Medical Co. LTD (SNUH 0620170600).

\section{CONFLICT OF INTEREST}

No potential conflict of interest relevant to this article was reported.

\section{AUTHOR CONTRIBUTIONS}

E.Y.L. had full access to all the data in the study and takes responsibility for the integrity of the data and the accuracy of the data analysis. J.M., J.S.L., Y.I.Y., S.H.C., Y.A.L., Y.J.H., E.H.K., Y.B.P., H.J.L., and J.Y.C. contributed substantially to the conception and design of the study, acquisition of data, analysis and/or interpretation of data, drafting the manuscript, and revising the manuscript, which is critically for important intellectual content.

\section{SUPPLEMENTARY DATA}

Supplementary data can be found with this article online at https://doi.org/10.4078/jrd.2021.28.4.234.

\section{REFERENCES}

1. Young A, Koduri G, Batley M, Kulinskaya E, Gough A, Norton S, et al. Mortality in rheumatoid arthritis. Increased in the early course of disease, in ischaemic heart disease and in pulmonary fibrosis. Rheumatology (Oxford) 2007;46: 350-7.

2. Bongartz T, Nannini C, Medina-Velasquez YF, Achenbach
SJ, Crowson CS, Ryu JH, et al. Incidence and mortality of interstitial lung disease in rheumatoid arthritis: a population-based study. Arthritis Rheum 2010;62:1583-91.

3. Olson AL, Swigris JJ, Sprunger DB, Fischer A, FernandezPerez ER, Solomon J, et al. Rheumatoid arthritis-interstitial lung disease-associated mortality. Am J Respir Crit Care Med 2011;183:372-8.

4. Gabbay E, Tarala R, Will R, Carroll G, Adler B, Cameron D, et al. Interstitial lung disease in recent onset rheumatoid arthritis. Am J Respir Crit Care Med 1997;156(2 Pt 1): 528-35.

5. Doyle TJ, Hunninghake GM, Rosas IO. Subclinical interstitial lung disease: why you should care. Am J Respir Crit Care Med 2012;185:1147-53.

6. Dawson JK, Fewins HE, Desmond J, Lynch MP, Graham DR. Predictors of progression of HRCT diagnosed fibrosing alveolitis in patients with rheumatoid arthritis. Ann Rheum Dis 2002;61:517-21.

7. Gochuico BR, Avila NA, Chow CK, Novero LJ, Wu HP, Ren $\mathrm{P}$, et al. Progressive preclinical interstitial lung disease in rheumatoid arthritis. Arch Intern Med 2008;168:159-66.

8. Saag KG, Teng GG, Patkar NM, Anuntiyo J, Finney C, Curtis JR, et al. American College of Rheumatology 2008 recommendations for the use of nonbiologic and biologic disease-modifying antirheumatic drugs in rheumatoid arthritis. Arthritis Rheum 2008;59:762-84.

9. Ascherman DP. Interstitial lung disease in rheumatoid arthritis. Curr Rheumatol Rep 2010;12:363-9.

10. Zangerle PF, De Groote D, Lopez M, Meuleman RJ, Vrindts Y, Fauchet F, et al. Direct stimulation of cytokines (IL-1 beta, TNF-alpha, IL-6, IL-2, IFN-gamma and GM-CSF) in whole blood: II. Application to rheumatoid arthritis and osteoarthritis. Cytokine 1992;4:568-75.

11. Ohnishi H, Yokoyama A, Kondo K, Hamada H, Abe M, Nishimura K, et al. Comparative study of KL-6, surfactant protein-A, surfactant protein-D, and monocyte chemoattractant protein-1 as serum markers for interstitial lung diseases. Am J Respir Crit Care Med 2002;165:378-81.

12. Kobayashi J, Kitamura S. KL-6: a serum marker for interstitial pneumonia. Chest 1995;108:311-5.

13. Hant FN, Ludwicka-Bradley A, Wang HJ, Li N, Elashoff R, Tashkin DP, et al. Surfactant protein D and KL-6 as serum biomarkers of interstitial lung disease in patients with scleroderma. J Rheumatol 2009;36:773-80.

14. Satoh $H$, Kurishima K, Ishikawa $H$, Ohtsuka M. Increased levels of KL- 6 and subsequent mortality in patients with interstitial lung diseases. J Intern Med 2006;260:429-34.

15. Lee JS, Lee EY, Ha YJ, Kang EH, Lee YJ, Song YW. Serum KL-6 levels reflect the severity of interstitial lung disease associated with connective tissue disease. Arthritis Res Ther 2019;21:58.

16. Kim HC, Choi KH, Jacob J, Song JW. Prognostic role of blood KL-6 in rheumatoid arthritis-associated interstitial lung disease. PLoS One 2020;15:e0229997.

17. Fotoh DS, Helal A, Rizk MS, Esaily HA. Serum Krebs von den Lungen- 6 and lung ultrasound $B$ lines as potential diagnostic and prognostic factors for rheumatoid arthritis-associated interstitial lung disease. Clin Rheumatol 2021 Jan 21 [Epub]. DOI:10.1007/s10067-021-05585-y.

18. Zheng M, Lou A, Zhang H, Zhu S, Yang M, Lai W. Serum KL-6, CA19-9, CA125 and CEA are diagnostic biomarkers 
for rheumatoid arthritis-associated interstitial lung disease in the Chinese population. Rheumatol Ther 2021;8:517-27.

19. Rosas IO, Richards TJ, Konishi K, Zhang Y, Gibson K, Lokshin AE, et al. MMP1 and MMP7 as potential peripheral blood biomarkers in idiopathic pulmonary fibrosis. PLoS Med 2008;5:e93.

20. White ES, Xia M, Murray S, Dyal R, Flaherty CM, Flaherty $\mathrm{KR}$, et al. Plasma surfactant protein-D, matrix metalloproteinase-7, and osteopontin index distinguishes idiopathic pulmonary fibrosis from other idiopathic interstitial pneumonias. Am J Respir Crit Care Med 2016;194:1242-51.

21. Chen J, Doyle TJ, Liu Y, Aggarwal R, Wang X, Shi Y, et al. Biomarkers of rheumatoid arthritis-associated interstitial lung disease. Arthritis Rheumatol 2015;67:28-38.

22. Doyle TJ, Patel AS, Hatabu H, Nishino M, Wu G, Osorio JC, et al. Detection of rheumatoid arthritis-interstitial lung disease is enhanced by serum biomarkers. Am J Respir Crit Care Med 2015;191:1403-12.

23. Hussein MS, El-Barbary AM, Nada DW, Gaber RA, Elkolaly RM, Aboelhawa MA. Identification of serum interleukin-13 and interleukin-13 receptor subunit expressions: rheumatoid arthritis-associated interstitial lung disease. Int J Rheum Dis 2021;24:591-8.

24. Minaur NJ, Jacoby RK, Cosh JA, Taylor G, Rasker JJ. Outcome after 40 years with rheumatoid arthritis: a prospective study of function, disease activity, and mortality. J Rheumatol Suppl 2004;69:3-8.
25. Kim D, Cho SK, Choi CB, Choe JY, Chung WT, Hong SJ, et al. Impact of interstitial lung disease on mortality of patients with rheumatoid arthritis. Rheumatol Int 2017;37:1735-45.

26. Kim JW, Lee H, Hwang JH, Park SH, Lee HS, Kim SK, et al. Factors associated with airway disease and interstitial lung disease in rheumatoid arthritis. J Rheum Dis 2016;23: 101-8.

27. De Lauretis A, Sestini P, Pantelidis P, Hoyles R, Hansell $\mathrm{DM}$, Goh NS, et al. Serum interleukin 6 is predictive of early functional decline and mortality in interstitial lung disease associated with systemic sclerosis. J Rheumatol 2013;40: 435-46.

28. Rincon M, Irvin CG. Role of IL-6 in asthma and other inflammatory pulmonary diseases. Int J Biol Sci 2012;8: 1281-90.

29. Furst DE, Emery P. Rheumatoid arthritis pathophysiology: update on emerging cytokine and cytokine-associated cell targets. Rheumatology (Oxford) 2014;53:1560-9.

30. Kinoshita F, Hamano H, Harada H, Kinoshita T, Igishi $T$, Hagino $\mathrm{H}$, et al. Role of KL-6 in evaluating the disease severity of rheumatoid lung disease: comparison with HRCT. Respir Med 2004;98:1131-7.

31. Richards TJ, Kaminski N, Baribaud F, Flavin S, Brodmerkel C, Horowitz D, et al. Peripheral blood proteins predict mortality in idiopathic pulmonary fibrosis. Am J Respir Crit Care Med 2012;185:67-76. 